\title{
Emanzipation oder Reaktion: Wie konservativ ist die deliberative Demokratie?
}

\author{
Andreas Schäfer • Wolfgang Merkel
}

Online publiziert: 19. März 2020

(C) Der/die Autor(en) 2020

Zusammenfassung Ist die deliberative Demokratie ein konservatives Modell? Beruht es auf Mechanismen, die in systematischer Weise seinen emanzipatorischen Anspruch unterminieren? Der Aufsatz beantwortet diese Frage im Anschluss an KritikerInnen der deliberativen Demokratie in Hinblick auf drei Dimensionen. Die zeitliche Dimension betreffend wird untersucht, inwieweit das deliberative Modell einen den Status quo bewahrenden Charakter hat. In der sachlichen Dimension wird eine Tendenz zur Entpolitisierung diskutiert. Für die soziale Dimension thematisiert der Aufsatz die potenzielle Exklusion bestimmter sozialer Gruppen und ihrer Perspektiven aus dem Deliberationsprozess. In Auseinandersetzung mit einschlägigen Ansätzen der deliberativen Demokratietheorie und mit Befunden der empirischen Deliberationsforschung gelangt der Aufsatz zu einem differenzierten Bild. Demnach lassen sich potenziell konservierende Tendenzen in der deliberativen Demokratie auffinden. Gleichzeitig wird argumentiert, dass diese Tendenzen vermeidbar sind, wenn einerseits das genuin kritische Potenzial deliberativer Praxis gegenüber anderen Elementen des Modells hervorgehoben und andererseits die Notwendigkeit der institutionellen Einbettung deliberativer Prozesse in Verfahren der repräsentativen Demokratie berücksichtigt wird.

Schlüsselwörter Inklusion · Exklusion · Ungleichheit · Systemische Wende · Demokratische Innovationen

\footnotetext{
A. Schäfer $(\bowtie)$

Humboldt-Universität zu Berlin, Unter den Linden 6, 10099 Berlin, Deutschland

E-Mail: andreas.schaefer.1@hu-berlin.de

W. Merkel

Wissenschaftszentrum Berlin für Sozialforschung, Reichpietschufer 50, 10785 Berlin, Deutschland E-Mail: wolfgang.merkel@wzb.eu
} 


\title{
Emancipation or Reaction: How Conservative is Deliberative Democracy?
}

\begin{abstract}
Is deliberative democracy a conservative model? Is it based on mechanisms that systematically undermine its emancipatory claim? Drawing on the work of critics of deliberative democracy, this essay answers those questions by considering three distinct dimensions of the problem. First, with regard to the temporal dimension, it examines the extent to which the deliberative model tends to uphold the status quo. Then, as far as the material dimension is concerned, it considers whether deliberative democracy encourages depoliticization. Finally, the essay investigates the social dimension: Are certain social groups and their perspectives potentially excluded from the deliberation process. By examing relevant approaches taken both by theorists of deliberative democracy and empirical research on its actual implementations, the authors reach some nuanced conclusions. While it is true that potentially conservative tendencies can be identified in deliberative democracy, those tendencies can be avoided in two ways. First, the genuinely critical potential of deliberative practice must be emphasized in preference to other elements of the model. Second, we must bear in mind that deliberative processes should be embedded institutionally in the procedures of representative democracy.
\end{abstract}

Keywords Inclusion · Exclusion · Inequality $\cdot$ Systemic turn · Democratic innovations

\section{Einleitung}

Der Aufsatz widmet sich einem paradoxen Phänomen. Die Theorien der deliberativen Demokratie verfolgen den Anspruch, ein politisch emanzipatorisches Modell zu liefern. Es soll das demokratische Versprechen auf gleiche politische Teilhabe und Selbstbestimmung aller BürgerInnen zu verwirklichen helfen und dabei den politischen Einfluss demokratisch nicht legitimierter sozialer Machtverhältnisse minimieren (u. a. Habermas 1994; Benhabib 1996; Cohen 1998; Dryzek 2000). Damit grenzen sich die deliberativen Demokratietheorien von aggregativen Demokratiemodellen ab, die aus ihrer Sicht in der politischen Wirklichkeit liberaler repräsentativer Demokratien vorherrschen (Habermas 1996, S. 277-283), aber tendenziell die in einer Gesellschaft vorhandenen Machtverhältnisse verstärken (Gutmann und Thompson 2004). Nicht Wahl oder Abstimmung allein garantiert im deliberativen Modell demokratische Legitimität, sondern vor allem die Qualität des vorausgehenden Diskussions- und Willensbildungsprozesses. Dieser soll so gestaltet sein, dass genau die aus ihm resultierenden ,,...] Regelungen Legitimität beanspruchen dürfen, denen alle möglicherweise Betroffenen als Teilnehmer an rationalen Diskursen zustimmen könnten“ (Habermas 1996, S. 299-300).

Dieser Anspruch hat früh Kritik hervorgerufen. Sie unterstellt dem deliberativen Modell antipluralistische und damit antidemokratische Effekte (Mouffe 2005, S. 108-128). Die politische Theorie und die Demokratieforschung stehen damit in Hinblick auf die Einschätzung der Potenziale und Probleme der deliberativen De- 
mokratie zunehmend vor einer paradoxen Situation. Während VerfechterInnen des deliberativen Demokratiemodells dieses für ein reformerisches, wenn nicht radikales (Bohmann 1998) oder gar revolutionäres (Fung 2005) politisches Ideal halten, behaupten Kritiker das Gegenteil: Das deliberative Ideal trage konservative oder antidemokratische Konnotationen (Sanders 1997, S. 348; Mouffe 2005), es sei in verhängnisvoller Weise blind für Machtverhältnisse und Interessenkonflikte in der Politik (Shapiro 1999, 2017). Deliberation bilde gar einen Mechanismus der ideologischen Beherrschung von WählerInnen, die aus diesem Grund nicht in ihrem besten Eigeninteresse Wahlentscheidungen treffen (Przeworski 1998).

Der vorliegende Aufsatz soll zur Klärung dieses Widerspruchs beitragen und spitzt das Problem auf die Frage zu: Ist die deliberative Demokratie ein - implizit konservatives Modell? Beruht das Modell auf Mechanismen, die in systematischer Weise das Gegenteil von dem bewirken, was Absicht und Anspruch ist? Obwohl die kritische Diskussion der deliberativen Demokratie schon in die dritte Dekade geht, sind viele grundsätzliche Fragen keineswegs systematisch geklärt und werden immer wieder in der Literatur aufgegriffen (siehe zuletzt u. a. Bächtiger et al. 2018; Elstub und McLaverty 2014; Flügel-Martinsen et al. 2014; Landwehr und Schmalz-Bruns 2014; Fishkin und Mansbridge 2017). Anlass zur erneuten Auseinandersetzung mit solchen Grundfragen bietet auch die „systemische Wende“ (Dryzek 2010, S. 6-8), die die deliberative Demokratietheorie schon vor dem programmatischen Artikel von Mansbridge et al. (2012) vollzogen hat und in der sie das Verhältnis von deliberativer Demokratie und bestehenden politischen Institutionen neu reflektiert.

Wir argumentieren in drei Schritten. Zunächst rekonstruieren wir die Kritik am deliberativen Modell unter den theoretischen Vorgaben des Vorwurfs des Konservatismus. Der Begriff des Konservatismus hilft uns dabei, die möglichen Einwände gegen den deliberativen Ansatz zu systematisieren und dabei auf solche Probleme zu fokussieren, die dessen expliziten (normativen) Erwartungen entgegenlaufen. Wir schlagen vor, ihn in drei interdependente Dimensionen aufzuschlüsseln, die sich systematisch auf das deliberative Modell beziehen lassen. Im zweiten Schritt überprüfen wir die theoretische und empirische Plausibilität dieser Perspektive: Wo trifft sie zu und wo nicht? Außerdem fragen wir nach den Implikationen der neueren Theorieentwicklung in Hinblick auf eine systemische Konzeption der deliberativen Demokratie für den vorliegenden Zusammenhang. Im dritten Schritt ziehen wir die Schlussfolgerungen aus der bisherigen Untersuchung und fragen, welche Aspekte des deliberativen Modells verändert oder betont werden müssten, um dem Vorwurf des Konservatismus zu entgehen und den Anspruch auf eine demokratisch-emanzipatorische Wirkung aufrechterhalten zu können.

\section{Dimensionen des Konservatismus}

Konservatismus ist ein unscharfer Begriff, der zur analytischen Verwendung einer genaueren Bestimmung bedarf (Vierhaus 1995). Schon seine Perspektive ist unklar: Während der traditionsorientierte Konservative den Status quo erhalten will, ist der Reformkonservative von der Notwendigkeit historischen Wandels überzeugt; der reaktionäre Konservative strebt danach, einen vergangenen Zustand wiederher- 
zustellen. Aussichtslos ist eine substanzielle Bestimmung. Die Ziele und Werte des Konservatismus bleiben mehrdeutig, variabel und schillernd (Greiffenhagen 2010). Am besten lassen sich der Begriff und das Phänomen des Konservatismus noch in seiner historischen Genese fassen, nämlich als Antwort auf den aufklärerischen und revolutionären Rationalismus und nicht zuletzt auf die Folgen der Französischen Revolution (Burke 1790). In diesem Sinne zeichnet sich der Konservatismus durch eine skeptische Haltung gegenüber theoretisch-konstruktivistischer Reflexion aus, betont den nicht artikulierbaren Wert gewachsener Institutionen ${ }^{1}$ und partikularer Lebensformen und verteidigt sie gegenüber programmatischen Neuerungen und universalistischen Ansprüchen (Hamilton 2016). Die zwei Minimalbedingungen für das Vorhandensein von Konservatismus wären also seine Verteidigungshaltung gegenüber einer drohenden Umwälzung oder tief greifenden Reform der sozialen Ordnung einschließlich ihrer bestehenden Privilegien und die damit verbundene politische Methodik der reaktiven und inkrementellen Anpassung (Müller 2006, S. 361-362).

Der Konservatismus ist also vor allem als Gegentendenz zum emanzipatorischen Rationalismus zu begreifen. Dahingegen richtet sich die normative Stoßrichtung der deliberativen Demokratie gegen die Übersetzung bestehender ökonomischer und sozialer Machtverhältnisse in politische und fordert die prinzipielle Möglichkeit der rationalen Zustimmung aller von politischen Entscheidungen Betroffenen ein (Habermas 1994). Damit fokussieren die deliberativen Überlegungen auf eine schmerzliche Lücke in den liberalen Demokratietheorien, die die politische Gleichheit meist nur formal konstitutionell einfordern, aber keine konkreten institutionellen Vorkehrungen dagegen treffen, dass sich die im Kapitalismus erzeugte sozioökonomische Ungleichheit in ungleiche politische Beteiligungs- und Repräsentationschancen umsetzt (Merkel 2014). Wenn wir im Folgenden also danach fragen, wie konservativ die deliberative Demokratie ist, zielen wir auf die Identifikation solcher Bausteine im theoretischen Modell selbst, die eine potenzielle Gegentendenz zu diesem demokratisch-emanzipatorischen Anspruch darstellen bzw. nicht intendiert hervorbringen.

Unter demokratischer Emanzipation verstehen wir die Erhöhung (oder zumindest Sicherung) der gleichen Möglichkeiten und der Effektivität politischer Selbstbestimmung aller BürgerInnen innerhalb eines sozialen Gemeinwesens. Konservatismus bedeutet dagegen die Schließung oder Umkehrung solch demokratischer Partizipation. Wir argumentieren, dass sich dieses analytische Konservatismuskonzept sinnvoll in drei Dimensionen (zeitlich, sachlich, sozial) aufschlüsseln lässt, die einerseits unterschiedliche Stränge der Kritik am Deliberationsmodell integrieren können und andererseits eine systematische Überprüfung auf Basis einer theoretischen und empirischen Evaluation erlauben. Sichtet man die deliberationskritische Literatur vor diesem Hintergrund, lässt sich der Konservatismus-Vorwurf entsprechend ordnen. Die zeitliche Dimension betreffend (Abschnitt ,Status-quo-Orientierung - Utopie als Konservatismus“) wird kritisiert, dass das deliberative Modell einen den Status quo bewahrenden Charakter habe, was angesichts der Zunahme demokratiegefährdender Entwicklungen wie wachsender sozioökonomischer und politischer Un-

\footnotetext{
1 In einem antirationalistischen Sinne wendet sich insbesondere F.A. von Hayek gegen die rationalistische Hybris, den gewachsenen Institutionen des Marktes ein „rationalistisches Design“ entgegenzustellen (Hayek 1971, S. 48).
} 
gleichheit (vgl. Crouch 2004; Mau und Schöneck 2015; Merkel 2015) sogar einem Rückschritt gleichkäme. In der sachlichen Dimension (Abschnitt „Entpolitisierung durch Rationalisierung“) wird eine Tendenz zur Entpolitisierung beklagt; bestimmte Entscheidungsmaterien werden so dem demokratischen Prozess entzogen. Für die soziale Dimension (Abschnitt „Deliberative Mechanismen politischer Exklusion“) identifiziert die Kritik schließlich eine Tendenz zur Exklusion bestimmter sozialer Gruppen aus dem Deliberationsprozess. Die drei Dimensionen stehen zwar in einem engen theoretischen wie praktisch-politischen Zusammenhang, die analytische Trennung erleichtert aber deren systematische Überprüfung.

Die hier unternommene kritische Evaluation vor dem Hintergrund des Konservatismusvorwurfs setzt eine Unterscheidung zwischen dem Ideal, dem Modell und der Praxis der deliberativen Demokratie voraus. Das Ideal beschreibt in abstrakter Weise einen normativ wünschenswerten politischen Zustand, ohne zu beanspruchen, dass dieser mit der tatsächlich beobachtbaren oder realisierten politischen Praxis übereinstimmt. Das Modell hingegen übersetzt normative Ansprüche in Vorgaben für die konkrete politische Praxis (z. B. in Form von Verfahrensvorgaben). Damit gehen wir davon aus, dass das Modell der deliberativen Demokratie entweder, soweit es beachtet wird, politische Praktiken bestimmt oder solche Praktiken rechtfertigt, soweit sie ihm entsprechen. Diese Praktiken könnten aber in systematischer Weise Ergebnisse hervorbringen, die den normativen Kriterien des Ideals widersprechen. Während das Ideal der deliberativen Demokratie seinem Anspruch nach klar als nichtkonservativ zu beurteilen ist, kann das jeweils konkretisierende Modell also durchaus konservative Implikationen haben und in Spannung mit den normativen Vorstellungen treten. Daher konzentriert sich die folgende Auseinandersetzung auf das Modell der deliberativen Demokratie und sein Verhältnis zur konkreten politischen Praxis. Sie bezieht sich also nur indirekt auf das dahinterstehende Ideal.

\subsection{Status-quo-Orientierung - Utopie als Konservatismus}

Von einem Demokratiemodell mit emanzipatorischem Anspruch müssten wir das Potenzial erwarten, illegitime soziale und politische Herrschaftsverhältnisse herauszufordern, aufzubrechen und zu transformieren. Der erste Vorwurf lautet jedoch, dass das deliberative Demokratiemodell nicht in der Lage sei, bestehende Verhältnisse infrage zu stellen. Es würde sogar dazu beitragen, Letztere aufrechtzuerhalten (Young 2001). Er betrifft die Dimension von Politik in und mit der Zeit. Emanzipation ist ein Prozessbegriff. Ihre Verwirklichung erfordert die Entfaltung einer zeitlichen Veränderung weg von einem als problematisch betrachteten Status quo hin zu einem präferierten Zustand. Diese Entwicklung kann in der politischen Auseinandersetzung durch entsprechende Institutionen, Strategien und Praktiken entweder gebremst oder befördert werden.

Eine frühe Kritik, die den Vorwurf der Status-quo-Fixiertheit begründet und explizit konservative Tendenzen des deliberativen Modells zu identifizieren beansprucht, stammt von Sanders (1997). Ihre Hauptthese ist, dass Deliberation zwar nicht an sich problematisch sei, sie aber angesichts vorherrschender widriger Umstände zwangsläufig bestehende Machtasymmetrien und Ungleichheiten nicht nur nicht überwinden, sondern festigen würde. Demnach könnte Deliberation nur dann ihr emanzi- 
patorisches Potenzial entfalten, wenn förderliche soziale Umstände existierten. Dies sei allerdings weder in der Gegenwart noch in absehbarer Zukunft zu erwarten. Die Erwartungen der deliberativen Demokratietheorie setzten also utopische Bedingungen voraus, deren Nichtexistenz die reale Wirkung von Deliberation in das Gegenteil ihrer Intentionen verkehrt. Wenn dieser Vorwurf zuträfe, würde das deliberative Modell dazu beitragen, bestehende soziale Machtverhältnisse in politische zu transformieren und zu konservieren.

Das Problem sei demnach, dass Deliberation bestehende Machtverhältnisse zum Ausdruck bringt und damit reproduziert. Dies sei der Fall, weil die Fähigkeiten zu deliberativer Partizipation von Ressourcen wie Zeit und Bildung abhängen, die analog zu ökonomischen Ressourcen ungleich verteilt sind. Deliberation könne daher nur bestehende Ungleichheitsverhältnisse stillschweigend bestätigen. Sie solle zwar partikulare Interessen für das Gemeinwohl transzendieren. Allerdings würden in der Regel damit bestimmte Interessen stillschweigend bedient (Status quo) und andere unterprivilegierte Interessen und Perspektiven benachteiligt.

Ein daran anschlussfähiger Vorwurf lautet, hegemoniale Diskurse würden die Sichtweisen und Argumente der TeilnehmerInnen an deliberativen Foren beeinflussen. Bestimmte unhinterfragte Vorstellungen, Kriterien und Bilder, die bestehende Machtverhältnisse widerspiegeln, steuerten auf subtile Weise die deliberativen Prozesse. So gesehen stünde Deliberation sogar in einem Spannungsverhältnis zu Formen des politischen Aktivismus, der für die Herstellung politischer Gleichheit und die Anfechtung sozialer Ungerechtigkeiten unter den gegenwärtigen Umständen Erfolg versprechender sei (Young 2001, S. 685-689).

\subsection{Entpolitisierung durch Rationalisierung}

Die sachliche Dimension betrifft die Frage, welche Inhalte und Positionen im deliberativen Prozess berücksichtigt werden. Gemäß seinem demokratisch-emanzipatorischen Anspruch müsste das deliberative Modell Möglichkeiten schaffen, sowohl alle Belange als auch alle Perspektiven von Entscheidungsbetroffenen effektiv in den politischen Prozess einzuspeisen. Die KritikerInnen beanstanden aber, dass sich das deliberative Modell nahtlos in bestehende Strukturen einpassen lässt. Die Gestaltung der Agenda sei üblicherweise nicht Gegenstand des deliberativen Prozesses, was die demokratische Autonomie der TeilnehmerInnen verletze (Urbinati 2010, S. 74-75). Deliberative Foren laufen daher Gefahr, zum Instrument von fremdbestimmter Selektion der politischen Entscheidungsmaterien zu werden.

Darüber hinaus bestünde die Gefahr, dass der Rationalitätsanspruch des deliberativen Modells der Bandbreite an legitimen Positionen und Argumenten im politischen Prozess zu enge Grenzen setzt. Die vermeintliche Konsensorientierung des deliberativen Modells wird dabei von KritikerInnen als eines der zentralen Mechanismen des Ausschlusses von Themen, Positionen und Argumenten dargestellt. Nach Mouffe (2007, S. 19) ignoriere die deliberative Demokratietheorie die Grundlogik des Politischen, die in dem Austragen antagonistischer Konflikte bestehe. Durch die falsche Annahme, dass ein rationaler Konsens auf Basis von fairen und neutralen Verfahren in klassengeprägten Gesellschaften möglich sei, würde in Wirklichkeit das Politische negiert. Tatsächlich sei aber ein sogenannter rationaler Konsens nur 
durch den Ausschluss anderer Sichtweisen, die als irrational eingestuft würden, herstellbar. Das Perfide dieser Strategie liege aber darin, dass diese Form von Exklusion nicht als eine politische, sondern eine moralische dargestellt werde. Da sie rational und moralisch begründet werde, sei sie weiterer legitimer Kritik entzogen.

Hinzu kommt der Vorwurf, das deliberative Modell würde seine theoretisch begründeten diskursiven Verfahren selbst der politischen Auseinandersetzung entziehen. Tully (2004, S. 96-98) argumentiert, dass ein rationaler Konsens über Standards fairer und gerechter Verfahren ebenso wenig erreichbar ist wie jener über andere politische Streitfragen. Für eine vollständige Demokratisierung der Willensbildung müssten auch deliberative Verfahren selbst als imperfekt erkannt werden und für eine ständige Infragestellung durch die beteiligten Akteure offen sein. Dem Vertrauen auf die rationalitätsbegründende Kraft deliberativer Verfahren, das sich bei Autoren wie Habermas findet, setzt er eine Skepsis entgegen, die die Möglichkeit einer prozessunabhängigen Bestimmung von fairen prozeduralen Regeln bezweifelt. Entsprechend müsse sich die Demokratietheorie in ihrem Anspruch beschränken und auf gleicher Ebene mit den politischen Akteuren in konkreten Konfliktfällen ihre Verfahrensvorschläge diskutieren.

Nach Urbinati (2010, S. 72-73) werden deliberative Foren gegenwärtig auch vor allem dazu eingesetzt, Konflikte durch die Herstellung eines unparteiischen Konsens zu entpolitisieren und somit den genuin demokratischen Institutionen der Wahl und Repräsentation zu entziehen (vgl. auch Shapiro 2017). Diese Rationalisierung der Demokratie(theorie) auf Kosten der demokratischen Partizipation (Buchstein und Jörke 2003) und zugunsten der Hervorbringung von vermeintlich überparteilichen Positionen bewirke dann letztlich die Schließung der politischen Agenda. Es kommt zu einer Entpolitisierung durch Rationalisierung.

\subsection{Deliberative Mechanismen politischer Exklusion}

Der Lackmustest des emanzipatorischen Potenzials deliberativer Demokratie ist die Frage nach der politischen Inklusion möglichst aller von kollektiven Entscheidungen Betroffenen. Die dritte Dimension des Konservatismusvorwurfs betrifft entsprechend die politische Exklusion bestimmter Schichten und Gruppen. Deliberative Settings würden in Top-down-Manier organisiert, sodass Eliten letztlich über die Einrichtung und Gestaltung deliberativer Verfahren bestimmten (Young 2001, S. 677-678). Sie werden damit zum Mittel politischer und administrativer Eliten, die durch allgemeine und gleiche Wahlen legitimierten Institutionen repräsentativer Demokratie zu umgehen oder in ihren Entscheidungen unter Druck zu setzen (Thaa 2007; Urbinati 2010, S. 74). Doch auch dort, wo die Zugangschancen für alle von einer Entscheidung Betroffenen formal gleich sind, sei der Zugang faktisch beschränkt, weil strukturelle Ungleichheiten solche Akteure begünstigten, die über mehr materielle, kognitive und soziale Ressourcen verfügten (Young 2001, S. 679-680). Am Ende entschieden wieder sozial privilegierte und ressourcenstarke Eliten über die Belange sozial ohnehin Unterprivilegierter (Shapiro 1999, 2017). Ein substanzieller Teil der BürgerInnen verlöre an Einfluss auf demokratische Entscheidungen, was, so spitzt Sanders zu, einem „disenfranchisement“ gleichkomme (Sanders 1997, S. 352). 
Doch neben der Ungleichverteilung der Zugangschancen ist es auch der Charakter von Deliberation selbst, der aus der Sicht der KritikerInnen Ausschlussrisiken in sich trägt. So betrachtet Young die sogenannte rationale Kommunikation nicht als universell gültige Kommunikationsform, sondern als einen schichtenspezifischen Stil der Auseinandersetzung (Young 2000; ähnlich Mouffe 2005). Durch den Ausschluss oder die Stigmatisierung alternativer politischer Artikulationsweisen (wie z.B. Storytelling) werden Menschen aus unterprivilegierten sozialen Schichten auch im Deliberationsprozess benachteiligt oder von ihm ferngehalten (Sanders 1997).

Gemäß Thaa (2007) bildet gerade die Informalisierung und Kognitivierung politischer Repräsentation einen problematischen Exklusionsmechanismus. Indem das Diskursprinzip ins Zentrum des Legitimationsgefüges gerückt wird, werde das formale Repräsentationsprinzip relativiert. Im Mittelpunkt stehe dann nicht mehr die Willensbeziehung zwischen Repräsentanten und Repräsentierten, über die auch Sanktionen erfolgen können, sondern die komplexe Unüberschaubarkeit von Verständigungsprozessen, die auf das politische Zentrum einwirken und eine Zurechenbarkeit politischer Entscheidungen erschweren. Die formelle Gleichheit bei der Wahl von Repräsentanten werde dann mittels Verlagerung der Legitimation auf Deliberationsprozesse informalisiert.

Im Ergebnis schließe das deliberative Modell viele aus der Partizipation und Dezision aus. Zwar würden deliberative Foren Partizipationsmöglichkeiten für eine ausgewählte Gruppe von BürgerInnen bereitstellen, sie würden aber gleichzeitig den verbleibenden Großteil der Menschen noch passiver machen (Urbinati 2010, S. 74). Darüber hinaus verleihen sie unter bestimmten Umständen den bestehenden Ausschlussverhältnissen Legitimität, indem sie ungewollt in „Komplizenschaft“ (Young 2001, S. 675) mit jenen Institutionen treten, die soziale und politische Ungleichheiten befördern.

\section{Der Konservatismusvorwurf in der Diskussion}

Wir haben also drei Dimensionen des Konservatismusvorwurfs identifiziert: Zeitlich bewirke das deliberative Modell die Erhaltung des Status quo struktureller Ungleichheit innerhalb einer Gesellschaft; sachlich schließe es die politische Agenda für bestimmte Themen, Positionen und Argumente, die eine sozial emanzipatorische Stoßrichtung haben könnten; und sozial führe es zu einer Exklusion bestimmter benachteiligter Bevölkerungsgruppen. Welche Antworten lassen sich nun in der deliberativen Demokratietheorie auf diese Kritikpunkte finden? Welche Schlüsse können wir dazu aus der empirischen Deliberationsforschung ziehen?

\subsection{Die zeitliche Dimension: Emanzipation vs. Status quo}

Klar ist, dass deliberative Verfahren ein entschleunigendes Moment in den demokratischen Prozess einbringen. Entscheidungen werden hierdurch dem unmittelbaren (vorläufigen) Mehrheitswillen entzogen und für einen verlängerten Zeitraum der kollektiven Reflexion zugänglich gemacht (Merkel und Schäfer 2015). Die dahinterstehende Annahme ist, dass die den Mehrheitsentscheidungen zugrunde liegenden 
Präferenzen immer prozessendogen und damit vom Verlauf von Deliberationsprozessen abhängig sind (Gutmann und Thompson 2004, S. 20). Deliberation lässt sich insofern als ein konservierendes Moment begreifen, als sie die demokratische Entscheidungsfindung entschleunigt und die Unmittelbarkeit der Abstimmung durch die BürgerInnen oder ihre demokratischen Repräsentanten verzögert. Gleichzeitig gehören solche temporalen Einschränkungen zum Wesen der Demokratie und können dazu dienen, Mehrheitsentscheidungen auch für die jeweilige Minderheit akzeptabel zu machen (Linz 1998). Entschleunigung kann so Vertrauen in die potenzielle Mitwirkungsmöglichkeit möglichst aller Herrschaftsunterworfenen herstellen.

Linz verweist allerdings auch auf eine Gefahr: Wird die demokratische Auseinandersetzung nicht klar befristet, gewinnen jene AktivistInnen an Macht, die über die größten zeitlichen Ressourcen verfügen, weil sich alle anderen aus Mangel an Zeit zunehmend vom Prozess abwenden (müssen), wodurch die demokratische Gleichheit in der Partizipation systematisch untergraben würde (Linz 1998, S. 26-27). Diese Überlegung ist auch auf deliberative Verfahren anwendbar. Ohne eine klare zeitliche Rahmung der Beratungszeit durch die Festsetzung eines Entscheidungszeitpunkts, an dem der Deliberationsprozess beendet bzw. unterbrochen wird, tritt der deliberative Prozess in ein Spannungsverhältnis zur Mitwirkungsgleichheit und führt potenziell entweder zum Stillstand oder dazu, dass am Ende diejenigen entscheiden, die die meiste Zeit haben.

Neben dem Umgang mit der Zeit bleibt die Frage nach den Erfolgen bzw. Hemmnissen demokratischer Emanzipation im Zeitverlauf. Entscheidend ist hierfür, ob die durch deliberative Verfahren zwischengeschaltete Beratungszeit auch Möglichkeiten der kritischen Infragestellung der herrschenden Verhältnisse schafft oder der Sicherung bestehender Machtverhältnisse dient. KritikerInnen betonen allerdings unter den Bedingungen gegenwärtiger gesellschaftlicher Machtverhältnisse die Gefahr der Manipulation von Sichtweisen auf die je eigenen Interessen und Werte von BürgerInnen im Verlaufe der Deliberation (vgl. Sanders 1997; Przeworski 1998).

VertreterInnen der deliberativen Demokratie sehen hingegen emanzipatorische Potenziale von Deliberation prinzipiell auch unter den bestehenden Verhältnissen heutiger repräsentativer Demokratien - ohne von einer Zwangsläufigkeit positiver Effekte auszugehen oder gegenüber möglichen negativen Konsequenzen blind zu sein. Auch angesichts augenfälliger Herausforderungen, die die stratifizierte und professionalisierte Struktur massenmedialer Kommunikation für rationale öffentliche Diskurse bereitstellen (vgl. Peters 2007, S. 190-194), entwirft etwa Habermas ein Forschungsprogramm, welches medialer politischer Kommunikation die spezifische Funktion zuschreibt, „reflektierte öffentliche Meinungen“ hervorzubringen, indem ,relevante Fragestellungen und kontroverse Antworten, erforderliche Informationen und geeignete Argumente für und wider mobilisiert werden" (Habermas 2008, S. 167). Öffentliche Deliberation findet dabei erklärtermaßen vor dem Hintergrund ungleicher politischer, sozialer und medialer Machtverhältnisse statt. Sie vermitteln einzelnen Akteuren strukturell höhere Chancen, den politischen Kommunikationsprozess in ihrem Sinne zu beeinflussen. Allerdings - so die Annahme finden diese ungleichen Einflussmöglichkeiten ihre Grenze in der reflexiven Struktur der Öffentlichkeit. Denn alle Einflussversuche müssen den Regeln des Gebens und Nehmens von Gründen, die mehr oder weniger Überzeugungskraft haben, gehor- 
chen (Habermas 2008, S. 173-179), wenn sie sich der kritischen Prüfung durch die Öffentlichkeit stellen. Voraussetzung für die Entfaltung dieser reflexiven Struktur ist jedoch die Unabhängigkeit des Mediensystems und eine Zivilgesellschaft, die BürgerInnen die Fähigkeit zur Partizipation an der öffentlichen Meinungsbildung verleiht. Vor diesem Hintergrund lassen sich dann entsprechende Pathologien identifizieren, wenn beispielsweise Medien in zu starke Abhängigkeit vom Staat oder vom Markt geraten oder wenn soziale und kulturelle Benachteiligungen den Zugang zur öffentlichen Meinungsbildung erschweren (Habermas 2008, S. 179-190).

Doch sind die in den Massenmedien bereitgestellten reflektierten Meinungen nur eine Voraussetzung für eine kritische Willensbildung. Hinzukommen muss die Kompetenz der BürgerInnen, diese Informationen kritisch zu verarbeiten und gegebenenfalls in aktive Stellungnahmen zu transformieren. Hier liefert die empirische Forschung widersprüchliche Ergebnisse. Nach Rosenberg (2014) unterstelle die deliberative Demokratietheorie BürgerInnen Kompetenzen, über die sie nicht verfügten: differenziert-analytisches, logisches und systematisches Denken, kritische Urteilskraft und Selbstreflexion sowie Empathie und Kooperationsfähigkeit. Unter Hinweis auf die sozial- und entwicklungspsychologische Forschung stellt er diese kognitiven, reflexiv-evaluativen und kommunikativen Fähigkeiten infrage. Die meisten Menschen tendierten demnach zu fehlerhaftem, konkretistischem Denken und logischen Kurzschlüssen, zu unkritischen, unreflektierten und präkonventionellen Urteilen sowie zu mangelnder Bereitschaft, andere und fremde Perspektiven anzuerkennen und sich mit ihnen konstruktiv auseinanderzusetzen (Rosenberg 2014, S. 101-106). Auch die deliberative Interaktion könne diese Tendenzen nur begrenzt entschärfen. Zwar könne gezeigt werden, dass TeilnehmerInnen in deliberativen Prozessen lernen, Informationen gewinnen, soziale Verbindungen aufbauen und auch ihre Meinungen ändern. Diese Änderungen blieben aber im Rahmen einer oberflächlichen, unkreativen und unkritischen Anpassung an soziale Normen und vorherrschende Machtverhältnisse. Deliberation würde in solchen Fällen eher als Mechanismus der sozialen Kontrolle dienen als der Kritik an bestehenden Verhältnissen und der darauf aufbauenden Emanzipation (Rosenberg 2014, S. 113).

Auch wenn man die vermeintlichen Anforderungen, die die deliberative Demokratietheorie nach Rosenberg an die kognitiven Kompetenzen von normalen DiskursteilnehmerInnen stellt, als zu anspruchsvoll betrachten kann, bleiben insbesondere die Einwände zur mangelnden kommunikativen Kompetenz für unsere Fragestellung relevant. Ob es allerdings eine angemessene Erwartung darstellt, dass DurchschnittsbürgerInnen objektiv, integriert und abstrahiert denken müssten, um die Komplexität sozialer Probleme zu erfassen und ihnen mit neuen Lösungsansätzen zu begegnen (Rosenberg 2014, S. 112), ist fraglich. Aus demokratietheoretischer Perspektive ist die Frage wichtiger, ob Deliberation BürgerInnen ein Instrument liefert, konkurrierende Angebote politischer Parteien und Eliten kritisch zu diskutieren und zu bewerten.

Hier liefern Experimente zum Einfluss von Framing durch politische Eliten positive Belege. Framingeffekten kann insofern ein manipulatives Potenzial zugeschrieben werden, als sie Haltungen durch die Art der Präsentation eines Sachverhalts und nicht durch überzeugende Gründe beeinflussen. Druckman und Nelson (2003) untersuchen, in welchen kommunikativen Kontexten sich Elitenframes auf die politischen 
Meinungen von EmpfängerInnen auswirkten. Sie zeigen, dass solche Framingeffekte vor allem auf VersuchsteilnehmerInnen feststellbar sind, die entweder dem Frame ohne anschließende deliberativ strukturierte Diskussion ${ }^{2}$ ausgesetzt sind oder aber in der anschließenden Diskussion in einer homogenen Gruppe nur Personen begegnen, die demselben Frame ausgesetzt wurden. Diskussionen in heterogenen Gruppen, deren TeilnehmerInnen unterschiedlichen Frames ausgesetzt wurden, bewirkten eine Neutralisierung der Framingwirkungen durch die Konfrontation mit alternativen Argumenten (Druckman und Nelson 2003, S. 737). Nach diesen Befunden kann Deliberation die Möglichkeiten von Eliten, BürgerInnen in ihrem Sinne zu manipulieren, einschränken, allerdings nur, wenn Letztere die Chance haben, in ihren kommunikativen Interaktionen gegenläufigen Argumenten zu begegnen.

In diesem Zusammenhang ist die Studie von Esterling et al. (2015) interessant. Sie analysiert am Fall von deliberativen Beteiligungsverfahren zur Gesundheitsreform in Kalifornien den Zusammenhang zwischen politischer Uneinigkeit und subjektiver Zufriedenheit mit der Qualität der deliberativen Veranstaltung. Die Befunde zeigen, dass TeilnehmerInnen die Qualität des Forums am höchsten einschätzen, wenn sie mit einem moderaten Ausmaß an politischer Uneinigkeit konfrontiert werden. Wenn BürgerInnen mit divergierenden Meinungen konfrontiert werden, bevorzugen sie die Situation moderater Diversität gegenüber gleichgesinnten Gruppen ebenso wie gegenüber polarisierten Diskussionen. Entscheidend für den deliberativen Erfolg sei, dass das institutionelle Design der Veranstaltung in den TeilnehmerInnen eine Neugier gegenüber moderater Uneinigkeit weckt, die zu argumentativer Auseinandersetzung anregt (Esterling et al. 2015, S. 544-545). Deliberativ strukturierte Gruppendiskussionen können nach diesen Befunden also zu einer (moderaten) politischen Mobilisierung beitragen.

Niemeyers (2011) Studie liefert schließlich explizit Belege für emanzipatorische Effekte durch die Teilnahme an deliberativen Mini-publics. Seine Untersuchung zweier Fallstudien zu Beteiligungsverfahren im Zusammenhang infrastrukturpolitischer Konflikte in zwei australischen Regionen zeigt, wie der deliberative Prozess TeilnehmerInnen dazu befähigte, sich von Einflüssen durch manipulative Berichterstattung und populistische öffentliche Rhetorik freizumachen. Die TeilnehmerInnen konnten sich im Prozess des deliberativen Verfahrens verschiedene Diskurse zur Beurteilung der Entscheidungsalternativen erarbeiten. Dies ermöglichte eine Neuordnung von Präferenzen sowie eine differenzierte und autonome Entscheidungsfindung (Niemeyer 2011, S. 124).

Die Erörterung des Potenzials deliberativer Prozesse für die Veränderung des Status quo liefert also ein differenziertes Bild. Zwar finden sich theoretische Argumente und empirische Befunde, die auf die Schwierigkeiten verweisen, vor die sich das deliberative Demokratiemodell angesichts der Strukturen massenmedial vermittelter Öffentlichkeit gestellt sehen. Die überwiegend negativen Einschätzungen der oben zitierten KritikerInnen müssen allerdings relativiert werden. Demnach kann es BürgerInnen selbst unter gegebenen Umständen gelingen, in öffentlichen Diskussionen

\footnotetext{
2 Diskussionsgruppen wurden minimal moderiert, sodass alle TeilnehmerInnen gleiche Möglichkeiten hatten, ihre Positionen zu artikulieren, ohne ihnen diesbezüglich einen Zwang aufzuerlegen (Druckman und Nelson 2003, S. 734).
} 
und deliberativen Prozessen ihre Wahrnehmungen und Präferenzen zu revidieren und für eine Veränderung des Status quo zu votieren. Die auch empirisch festgestellte Möglichkeit der Präferenzänderung spricht in der zeitlichen Dimension gegen den Vorwurf grundsätzlicher Konservierung des Status quo.

\subsection{Die sachliche Dimension: Demokratisierung vs. Rationalisierung?}

In Hinblick auf den in Abschnitt „Entpolitisierung durch Rationalisierung“ rekonstruierten Vorwurf einer Entpolitisierungstendenz lässt sich bei häufig anzutreffenden Lesarten der deliberativen Demokratie tatsächlich zum Teil eine rationalistischformalistische Engführung von Deliberation ausmachen. Diese Engführung erweist sich aber mit Blick auf die theorieinterne Diskussion sowie auf empirische Forschungsbefunde als nicht zwingend. Sie bezieht sich zum einen auf die Form und zum anderen auf das Ergebnis von Deliberation. So erweist sich die verbreitete Annahme, Deliberation müsse stets in einer rational-unparteiischen Form durchgeführt sowie mit einem Konsens beschlossen werden, wenn sie legitimierende Kraft entfalten soll, bei genauerer Betrachtung als unzutreffend.

Beide Aspekte finden sich in pointierter Form aber bereits bei Elster (1998), dessen Interpretation die Sichtweise auf das deliberative Modell nachhaltig geprägt hat. Elster führt aus: „Reason is impartial, both disinterested and dispassionate. Arguing is intrinsically connected to reason, in the sense that anyone who engages in argument must appeal to impartial values“ (Elster 1998, S. 6). Elster fährt fort, dass nach übereinstimmender Sichtweise ihrer VertreterInnen zur deliberativen Demokratie gehöre, , ,[...] that it includes decision making by means of arguments offered by and to participants who are committed to the values of rationality and impartiality" (Elster 1998, S. 8). Ein solcher Deliberationsbegriff zeichnet sich also durch Nichtinteressiertheit, Emotionslosigkeit sowie Unparteilichkeit der Diskursbeteiligten sowie ihrer Diskurspraxis aus.

Gegen diese Auffassung von Deliberation hatten allerdings schon Gutmann und Thompson (1996) argumentiert. Demnach habe als das zentrale Prinzip der deliberativen Demokratie die Reziprozität zu gelten, das die AutorInnen von jenem der Unparteilichkeit klar abgrenzen. Zwar müsse auch Reziprozität darauf abzielen, allgemein akzeptable Gründe für die jeweiligen Positionen zu liefern. Anders als Unparteilichkeit fordere Reziprozität aber nicht, dass Gründe unabhängig von der Person und parteiischen Perspektiven sind (Gutmann und Thompson 1996, S. 53-54). Dementsprechend könne Deliberation auch nicht den Charakter einer wahrheitsfähigen Beweisführung haben, weil eine solche vor dem Hintergrund konkurrierender Wertvorstellungen in pluralistischen Gesellschaften nicht erreicht werden könne. Stattdessen ist Deliberation eine genuin politische Form der Auseinandersetzung mittels wechselseitiger Begründungsbemühungen. Diese zielt zwar auf Akzeptanz, erträgt aber durchaus Dissens, solange der Prozess fair erscheint.

Wie sieht es vor diesem Hintergrund mit dem Einwand aus, das deliberative Modell schlösse Kommunikationsformen als irrational aus, die nicht den Standards kühl-rationaler und unparteiischer Argumentation gerecht werden? Die theorieinterne Diskussion bestätigt dies nicht. So entwickelt Mansbridge die von Gutmann und Thompson vorgebrachten Überlegungen weiter. Sie verweist dabei auf die Notwen- 
digkeit, im deliberativen Modell nicht nur im engeren Sinne politische Arenen in den Blick zu nehmen, sondern auch jene alltäglichen Kommunikationsformen unter BürgerInnen, die die politische Entscheidungsfindung indirekt beeinflussen. Diese Formen müssten zwar prinzipiell die gleichen normativen Standards der Deliberation - wie etwa Reziprozität - in den anderen Kommunikationsarenen teilen, können aber in einer informelleren Kommunikationssituation auch gelockert werden. Mansbridge argumentiert: ,In both legislative bodies and the rest of the deliberative system the concept of ,public reason" should be enlarged to encompass a ,considered " mixture of emotion and reason rather than pure rationality“ (Mansbridge 1999, S. 213; siehe auch Chambers 2004).

Auch die empirische Deliberationsforschung hat die Kritik am vermeintlichen Ausschluss spezifischer Kommunikationsformen in ihren Vorschlägen zur Operationalisierung der demokratietheoretischen Annahmen produktiv aufgenommen. Bächtiger et al. (2010) schlagen beispielsweise vor, alternative Kommunikationsformen wie Storytelling zu berücksichtigen. Diesen wird nun eine phasenspezifische Funktionalität innerhalb des deliberativen Prozesses zugesprochen. Hierzu liefern Polletta und Lee (2006) weitere Erkenntnisse. Sie konzentrieren sich in ihrer empirischen Untersuchung von deliberativen Onlineforen, die zur Frage der Neugestaltung des Ground Zero unter ausgewählten Einwohnern von New York City durchgeführt wurden, auf die Rolle von Storytelling im Vergleich zu formaler Argumentation in Form von „reason-giving“. Die AutorInnen gehen von der Annahme aus, dass in deliberativen Kontexten Geschichten erzählt werden, um eine Haltung zu begründen. Es geht also nicht nur um das Erzählen, sondern darum „to make a point“ (Polletta und Lee 2006, S. 702).

Polletta und Lee zeigen mit ihrer Untersuchung auf, dass Storytelling nicht nur nicht im Widerspruch zu stärker formalisierter Argumentation - im Sinne von ,reason-giving“ - treten muss, sondern im Gegenteil auch wichtige deliberative Funktionen erfüllen kann. Storytelling teilt bestimmte strukturelle Merkmale von ,,reasongiving“ (Polletta und Lee 2006, S. 699-705), bringt diese aber anders zum Ausdruck und erzielt auf diese Weise komplementäre Diskursleistungen. Empirisch können die AutorInnen demonstrieren, dass die ForenteilnehmerInnen besonders häufig von Storytelling Gebrauch machen, wenn sie eine Sichtweise deutlich machen wollen, die sie als marginalisiert wahrnehmen, um ihr dadurch Plausibilität und Geltung zu verleihen (Poletta und Lee 2006, S. 711). Damit zielen sie auf eine Infragestellung vermeintlich universeller und neutraler Prinzipien, die in der gängigen Argumentation zum Thema dominieren. Dadurch eröffnen sich Chancen, neue Themen und Sichtweisen auf die kollektive Entscheidungsagenda zu platzieren.

Polletta und Lee arbeiten allerdings auch die Grenzen heraus, die den positiven Wirkungen von Storytelling gesetzt sind. Offenbar durch kulturell festgeschriebene Bewertungshierarchien wird Storytelling von den beteiligten Akteuren selbst als ambivalente Kommunikationsform mit einer seriösen und einer unseriösen Seite betrachtet. Dadurch wird der Angemessenheitsrahmen auf ein engeres Themenfeld eingeschränkt: Je mehr es um konkrete Policyentscheidungen geht, desto stärker nimmt der Rückgriff auf Geschichten ab und jener auf formales Argumentieren zu: „Narrative claims were not ignored altogether, but they did lose rhetorical force in such discussions“ (Polletta und Lee 2006, S. 716). Dieser wichtige Punkt macht 
deutlich, dass trotz guten Willens von Facilitatoren, tief eingeprägte kulturelle Muster nicht verschwinden, sondern dazu beitragen, Kommunikation zu stratifizieren. Dabei bleibt die besondere Gefahr bestehen, dass die kulturell tradierten Wertigkeiten vor allem bei sozialen Gruppen greifen, die ohnehin unterprivilegiert sind.

Der zweite Aspekt der konzeptuellen Engführung in der Kritik betrifft die Entscheidungsfindung innerhalb von Deliberation. Elster etwa behauptet, dass die Idee der deliberativen Demokratie in der Entscheidungsfindung mittels Diskussion (Elster 1998, S. 1) liege. Er interpretiert damit Deliberation primär als einen Entscheidungsmechanismus neben anderen Formen des Entscheidens wie Verhandeln oder Abstimmen (Elster 1998, S. 5). Diese Auffassung ist zu pauschal und nicht haltbar. Gutmann und Thompson (2004, S. 18) stellen beispielsweise klar, dass deliberative Demokratie keinen ,natürlichen“ Mechanismus der Entscheidungsfindung bereitstellt, wenn es keinen Konsens gibt, der in der Regel auch nicht zu erwarten sei. Deshalb müsse Deliberation immer mit anderen Entscheidungsprozeduren kombiniert werden. ${ }^{3}$

Die Annahme, Deliberation sei ein Modus des kollektiven Entscheidens setzt offenbar implizit voraus, dass Deliberation zwangsläufig auf einen rationalen Konsens hinausläuft, der wiederum eine andere Art des Entscheidens überflüssig macht. Dies ist aber weder eine realistische noch theoretisch notwendige Annahme. Genau an dieser Stelle setzen aber, wie wir oben gesehen haben, KritikerInnen wie Chantal Mouffe an und werfen dem deliberativen Modell eine entpolitisierende Tendenz vor. Denn dominiert die Konsensforderung, dann kann sie dazu dienen, bestehende Konflikte durch die Rationalitätsforderung stillzustellen.

Eine Anerkennung der Verschiedenheit von Deliberation als diskursiver Praxis und konsensueller Entscheidung erlaubt uns dagegen, sowohl die Befürchtungen der KritikerInnen ernst zu nehmen als auch die modernen Auffassungen der deliberativen Demokratie von älteren konservativen Vorstellungen abzugrenzen, wie sie etwa bei Burke als rationale Einigung unter vernünftigen Eliten verstanden wird. Neuere Ansätze der theoriegeleiteten Deliberationsforschung haben aufgezeigt, wie in konkreten Untersuchungskontexten einer solchen Engführung zu entkommen ist, indem der jeweils spezifische demokratietheoretische Wert von parteiischen (White und Ypi 2011), kontestativen (Schäfer 2017b) oder advokatorischen (Urbinati 2000) Formen von Deliberation hervorgehoben wird. Pragmatischere Operationalisierungen, die aber dennoch den normativen Kern einfangen (vgl. Fishkin und Luskin 2005, S. 285), erlauben dann jenseits einer formalistischen Orthodoxie, sowohl Potenziale als auch Pathologien des deliberativen Modells zu erfassen.

\subsection{Die soziale Dimension: Inklusion vs. Exklusion?}

Der normative Anspruch des deliberativen Modells ist klar: Alle Entscheidungsbetroffenen bzw. Herrschaftsunterworfenen sollen die möglichst gleiche und unbe-

\footnotetext{
3 Auch Benhabib (1996, S. 72) und Habermas (1990, S. 42) heben den funktional komplementären Zusammenhang zwischen Deliberation und Mehrheitsentscheid hervor. Vgl. auch Niesen (2014), der ideengeschichtlich belegt, dass Deliberation ein zwar entscheidungsbezogenes Konzept, aber kein Modus des Entscheidens ist.
} 
schränkte Chance haben, an politischen Willensbildungsprozessen teilzuhaben und aus freien Stücken kollektiv verbindliche Entscheidungen zu befürworten oder abzulehnen (Cohen 1998, S. 19; Benhabib 1996, S. 68; Habermas 1996, S. 299-300; Gutmann und Thompson 2004, S. 48, 50). Doch kann dieser Anspruch angesichts bestehender gesellschaftlicher Ungleichheiten durchgesetzt werden? Dieser Sachverhalt ist auf zwei Ebenen zu betrachten. Auf der ersten geht es um die Frage, wie inklusiv der Zugang zu deliberativen Verfahren bzw. Partizipationsmöglichkeiten ist. Auf der zweiten ist danach zu fragen, ob TeilnehmerInnen durch spezifisch deliberative Mechanismen innerhalb des Verfahrens systematisch benachteiligt werden.

Konzentrieren wir uns zunächst auf die Zugangsebene: Wer nimmt eigentlich an deliberativen Prozessen teil? Sind es nur die ökonomisch, kulturell und politisch Privilegierten, wie die oben zitierten KritikerInnen behaupten, oder können wir mit einer gleichmäßigen Partizipation über soziale Klassen, Schichten, Geschlechter und Milieus hinweg rechnen? Cook et al. (2007) liefern Befunde für die Verteilung von diskursiver Partizipation in den USA, die eine eher positive Sicht nahelegen. In ihrer Studie überprüfen sie anhand von repräsentativen Umfragedaten, inwiefern die einschlägigen sozioökonomischen und kulturellen Ungleichheiten (z.B. Bildung) die diskursive Partizipation von BürgerInnen beeinflussen. Im Ergebnis finden sich zwar Hinweise auf den Einfluss verzerrender Faktoren; diese fielen für diskursive Partizipation aber deutlich geringer aus als für andere partizipative Formen wie z. B. Wahlen. Wichtiger als Bildung erweise sich zudem die Mitgliedschaft in Organisationen und das politische Interesse. Dies ist ein Befund, der zumindest nahelegt, dass durch die Schaffung von diskursiven partizipativen Gelegenheitsstrukturen egalitäre Partizipationsmuster etabliert werden können (Cook et al. 2007, S. 41-42).

Andere Forschungsergebnisse weisen in eine ähnliche Richtung. Neblo et al. (2010) wenden sich dagegen, tatsächliches Partizipationsverhalten unter gegenwärtigen Bedingungen als alleinigen Maßstab für die Umsetzbarkeit deliberativer Demokratie zu bewerten. Man müsse auch ihre gegenwartskritische und zukunftsgewandte Stoßrichtung berïcksichtigen. Die Autoren liefern Belege für die These, dass bei BürgerInnen ein grundsätzlicher Bedarf bestehe, deliberative Partizipationsformen stärker zu nutzen. Zudem würden gerade Menschen aus jenen Gruppen von Deliberationsgelegenheiten angesprochen werden, die sich eher vom politischen Engagement in Parteien und Verbänden abgewandt haben (Neblo et al. 2010, S. 269). So zeigten sich in Befragungen im Vergleich zu traditionellen Partizipationsformen überdurchschnittlich viele nichtweiße, jüngere und geringverdienende Personen offen für deliberative Partizipationsformen (Neblo et al. 2010, S. 574). Auch diejenigen Befragten, die in der Untersuchung tatsächlich das Angebot zur Teilnahme an einem deliberativen Forum annahmen, erwiesen sich nicht als die üblichen Verdächtigen aus der Gruppe von AktivistInnen, die auch überdurchschnittlich oft in anderen Partizipationsformen zu finden sind. Ähnlich argumentieren auch Pogrebinschi und Samuels in ihrer Untersuchung zu den „National Public Policy Conferences“ in Brasilien (Pogrebinschi und Samuels 2014). Beide AutorInnengruppen schließen daraus, dass viele demobilisierte BürgerInnen sich dem politischen Prozess zuwenden würden, wenn klassische Partizipationsformen in deliberative Rahmenbedingungen „eingebettet“ wären (Neblo et al. 2010, S. 582). 
Solche Befunde legen nahe, dass der Zugang zu und die Motivation zur Partizipation an deliberativen Verfahren und Diskussionen im Allgemeinen nicht das gravierendste Exklusionsproblem darstellen. Dieses empirische Argument zur Teilnahmebereitschaft belegt aber noch nicht, dass Deliberation ein Mittel sein könnte, die Bedingungen von demokratischen Willensbildungs- und Entscheidungsprozessen vor dem Hintergrund sozialer Ungleichheiten selbst zu verbessern. Der Erfolg von Deliberation im Sinne eines emanzipatorischen Instruments wäre noch zu oft abhängig vom Wohlwollen politischer Eliten, die deliberative Partizipationsformen in bestimmten Situationen organisieren (vgl. McLaverty 2014). Schon aus diesem Grund wäre die deliberative Demokratietheorie gut beraten, das demokratische Potenzial von Deliberation nicht nur und nicht einmal in erster Linie in veranstalteten Mini-publics zu suchen (vgl. Chambers 2009; Lafont 2015).

In der zweiten Dimension der Inklusionsproblematik überprüft die empirische Forschung, ob die expliziten wie impliziten Regeln des Diskurses unterprivilegierte Gruppen gegenüber dominierenden benachteiligen, indem sie unter anderem die Verteilung von Redeanteilen in deliberativen Arenen untersucht. Gerber (2015) liefert Belege für die Wirksamkeit informeller Exklusionsmechanismen in einer Untersuchung von Kleingruppendiskussionen innerhalb eines europaweiten deliberative polls. Im Ergebnis zeigt sich, dass Frauen, TeilnehmerInnen aus neuen EUMitgliedsstaaten oder aus der Arbeiterklasse signifikant geringere Redeanteile aufweisen (Gerber 2015, S. 125-126). Karpowitz et al. (2012) untersuchen die Exklusionsdynamik dahingehend, ob Frauen in ihrem Partizipationsverhalten sowie in der Beachtung ihrer Beiträge in deliberativen Foren schlechter gestellt sind als männliche Teilnehmer. Mittels eines experimentellen Forschungsdesigns weisen die AutorInnen nach, dass es in gemischten Diskussionsgruppen in der Regel einen Gender Bias gibt und Frauen signifikant geringere Redeanteile aufweisen (Karpowitz et al. 2012, S. 545). Gleichzeitig zeigen die AutorInnen aber auch, dass die genannten Ungleichheitseffekte von der jeweiligen Gruppenzusammensetzung und der Entscheidungsregel abhängen. Sie weisen darauf hin, dass bei einer weiblichen Minderheit die Einstimmigkeitsregel und bei einer (deutlichen) weiblichen Mehrheit die Mehrheitsregel dazu beiträgt, den Gender Bias zu neutralisieren (Karpowitz et al. 2012, S. 538-543; vgl. auch Mendelberg et al. 2014). Die AutorInnen folgern: „The fact that gender inequality disappears under some conditions means that deliberation can in fact meet the standard of equality, as its advocates contend" (Karpowitz et al. 2012, S. 545).

Deliberation kann, muss aber nicht soziale Exklusion verstärken. Dabei sind es nicht in erster Linie deliberative Verhaltensweisen oder -mechanismen selbst, sondern kulturell imprägnierte Rollenmuster, Normen und Bewertungssysteme, die strukturelle soziale Ungleichheiten stabilisieren und emanzipatorische Potenziale deliberativer Partizipation unterminieren. Es gibt aber ganz offenbar Möglichkeiten, diese Effekte durch bestimmte institutionelle Designs zu neutralisieren. So belegen weitere Studien die Möglichkeit, durch explizite Regeln für die mediale Berichterstattung die besondere Berücksichtigung marginalisierter Gruppen in öffentlichen politischen Debatten zu sichern (Curato und Ong 2014). Darüber hinaus können benachteiligte Gruppen durch die Teilnahme an deliberativen Verfahren ihr politisches Wissen erhöhen (Fraile 2014). 


\section{Implikationen der systemischen Wende}

Welche Implikationen hat die „systemische Wende“ (Dryzek 2010, S. 7) für die „Konservatismusfalle“? Das Nachdenken über den systemischen Zusammenhang unterschiedlicher deliberativer Arenen und Prozesse innerhalb politischer Systeme ist in der Demokratietheorie keineswegs neu (vgl. Habermas 1994); es hat in den letzten Jahren nur deutlich an Dynamik gewonnen. Dryzek (2010, S. 11-12) unterscheidet mit „public space“, „,empowered space“, „transmission“, ,,accountability“, „meta-deliberation“ und „decisiveness“ sechs zentrale Dimensionen eines deliberativen Systems. Zentral ist dabei das komplementäre Ineinandergreifen verschieden verfasster deliberativer Arenen mit unterschiedlichen Kompetenzen (vgl. auch Mansbridge et al. 2012). Anstatt einzelne deliberative Foren wie Mini-publics oder Parlamente und deren jeweilige diskursive Qualität zu betrachten, sollten deren Interdependenzen daraufhin untersucht werden, wie ein deliberatives ,System" unterschiedlicher, aber komplementärer Arenen zu schaffen sei. Eine wichtige Implikation dieses systemischen Denkens ist, dass auch (vermeintlich) nichtdeliberative Mechanismen deliberative Funktionen erfüllen können (Mansbridge et al. 2012, S. 3).

Systemisches Denken erschwert eine kritische Evaluation des deliberativen Modells aufgrund des Arguments, dass deliberative Schwächen an einer Stelle des Systems durch Stärken an anderer Stelle ausgeglichen werden könnten; oder umgekehrt, dass Einschränkungen hinsichtlich des deliberativen Anspruchs in bestimmten Phasen des politischen Prozesses notwendig sind, um insgesamt den demokratischinklusiven Charakter des Systems zu gewährleisten. Einzelne Momente können dann nicht mehr unabhängig von ihrer potenziellen Interaktion bewertet werden, was die Evaluation verkompliziert, weil hierfür immer die Systemleistung als ganze berücksichtigt werden muss.

Doch muss wegen dieser Herausforderung eine Evaluation systemischer Modelle nicht aufgegeben werden. Die von uns vorgeschlagenen drei Dimensionen des Konservatismusvorwurfs können auch hier einen analytischen Rahmen liefern. Fragen wir also, ob ein systemisches Modell deliberativer Demokratie dazu beiträgt, den politischen Status quo zu konservieren, durch Rationalisierung Konflikte zu entpolitisieren und bestimmte soziale Gruppen aus dem Willensbildungs- und Entscheidungsprozess auszuschließen, lassen sich die entsprechenden potenziellen Vorund Nachteile identifizieren. Nachteilig würde sich die systemische Wende dann auswirken, wenn mit ihr jegliche Kritik am Status quo der gegenwärtigen Praktiken und Institutionen demokratischer Willensbildung und Entscheidungsfindung ins Leere laufen würde, weil auch noch die größte Abweichung vom Ideal als funktional und daher notwendig für das Erreichen des deliberativen Gesamtsystems gerechtfertigt werden kann. Damit liefe das deliberative Modell Gefahr, sein normativkritisches Potenzial zu verlieren und zur Legitimation gebräuchlicher, aber demokratietheoretisch problematischer kommunikativer Praktiken beizutragen (Owen und Smith 2015, S. 223). Daher muss mit jedem „trade-off“ eine kritische Überprüfung einhergehen, ob die Gesamtbilanz an deliberativ-demokratischen Werten insgesamt gesteigert wird oder abnimmt (Goodin 2018). 
Problematisch wäre ferner, wenn der systemische Charakter des deliberativen Modells die Möglichkeiten über das notwendige Maß hinaus vervielfältigen würde, potenziell konflikthafte politische Entscheidungsmaterien der demokratischen Auseinandersetzung zu entziehen, indem sie beispielsweise komplett in Expertengremien oder non-majoritäre Arenen verlagert werden. Auf diese Weise würde eine fragwürdige Arbeitsteilung zwischen elitär-deliberativen und populär-demokratischen Prozessen Platz greifen. Dies würde das demokratisierende Potenzial deliberativer Partizipation untergraben. Schließlich würde drittens das systemische Modell das Emanzipationspotenzial der deliberativen Demokratie einschränken, wenn es exkludierende Mechanismen der Deliberation - wie beispielsweise vorherrschende kulturelle Hierarchien innerhalb von kommunikativen Praktiken - verstärken würde. Dies könnte beispielsweise dadurch zustande kommen, dass die Bandbreite an legitimen Formen der diskursiv-demokratischen Auseinandersetzung vonseiten der Demokratietheorie, der Leitmedien oder der vorherrschenden Meinungsführer zu eng gefasst wird oder sich zu einseitig an Kommunikationsstandards exklusiver kultureller Milieus orientiert, ohne dass die kulturelle Prägung und Stabilisierung sozialer Stratifizierung berücksichtigt wird.

Tritt man mit diesen kritischen Fragen und Erwägungen an das systemische Modell deliberativer Demokratie heran, treten aber auch dessen Vorteile und Chancen deutlich hervor. Die systemische Perspektive bietet erstens eine Abkehr vom (exklusiven) Fokus auf Mini-publics, deren Effektivität oft genug von der wohlwollenden Berücksichtigung durch politische Eliten abhängt. Sie fokussiert auf die Verbindung unterschiedlicher Arenen der Willensbildung und Entscheidungsfindung und dabei besonders auf die Transmission von BürgerInnenpartizipation in politische Entscheidungen (vgl. Boswell et al. 2016; Hendriks 2016). Zweitens legt die systemische Perspektive eine Vermeidung der oben diskutierten formalistischen Orthodoxie nahe, die eine rationalistische Engführung des Deliberationskonzepts impliziert. Letztere begünstigt damit unbeabsichtigt kulturell imprägnierte Bewertungssysteme zulasten benachteiligter Gruppen und unterminiert die emanzipatorische Wirkung deliberativer Praktiken. Der systemische Zugang erkennt dagegen den demokratietheoretischen Wert von nichtdeliberativen Institutionen und Praktiken an und akzeptiert unterschiedliche Formen von Deliberation - seien sie formal-argumentativ, persönlicherzählerisch oder rhetorisch-kontestativ. Insbesondere kontestative Formen der Deliberation können das Potenzial haben, einen durch soziale Ungleichheiten geprägten Status quo in der öffentlichen Auseinandersetzung herauszufordern. Zusätzlich können persönlich-erzählerische Deliberationsformen (Storytelling) eine wichtige Rolle bei der Klärung und Artikulation von peripheren oder marginalisierten Präferenzen und Perspektiven spielen. Die Frage ist dann weniger, was ein System als Ganzes deliberativ macht, sondern welche Rolle Deliberation neben anderen Praktiken und Institutionen innerhalb des demokratischen Systems spielt (Owen und Smith 2015, S. 232). Auf diese Weise kann das demokratisch-emanzipatorische Potenzial deliberativer Praktiken realisiert werden, ohne dass der partizipative Wert anderer demokratischer Institutionen geschmälert wird. Unterschiedliche Institutionen und Praktiken können verschiedene demokratische Leistungen unterschiedlich gut erbringen (vgl. Warren 2017). Während gelingende Deliberation sich beispielsweise gut dazu eignet, komplexe Willensbildungsprozesse differenziert und fair zu gestal- 
ten, können Wahlen und Abstimmungen Stimmen gleich verteilen und Entscheidungen effektiv gestalten. Systemisches Denken sensibilisiert für die notwendigen Feinabstimmungen im Verhältnis dieser unterschiedlichen demokratischen Institutionen, die oftmals nur in Kombination dazu beitragen können, demokratisierende Potenziale zu entfalten. Diese Reflexionen über die Funktionsweisen des deliberativen Systems müssen aus Sicht der jüngeren Diskussion keineswegs, wie einst Tully (2004) kritisierte, alleinige Aufgabe der politischen Theorie und etablierten Verfassungspolitik bleiben. Metadeliberation, verstanden als die kritische Auseinandersetzung um die Art und Weise, wie deliberative Prozesse unter gegenwärtigen Bedingungen vonstattengehen, kann und muss Teil eines inklusiven und insgesamt diskursiven Willensbildungsprozesses innerhalb deliberativer Systeme sein (vgl. Dryzek 2010; Landwehr 2015; Holdo 2019).

Eine umfassende Evaluation von Konservatismus- vs. Emanzipationspotenzialen des systemischen Modells deliberativer Demokratie kann und soll an dieser Stelle nicht geleistet werden. Hierfür wäre auch die Auseinandersetzung mit konkreten Realtypen deliberativer Systeme notwendig. Die selektive Diskussion zeigt aber, dass systemisches Denken in der deliberativen Demokratietheorie trotz dessen methodischer Herausforderungen konzeptuelle Mittel bereithält, emanzipatorische Antworten auf den Konservatismusvorwurf zu finden.

\section{Fazit}

Ist die deliberative Demokratie ein konservatives Modell? Produziert sie Mechanismen, die in systematischer Weise das Gegenteil von dem bewirken, was ihr Anspruch ist? Diese Frage hatten wir auf drei Dimensionen des Konservatismusvorwurfs heruntergebrochen: Das deliberative Modell unterstütze den politischen Status quo, führe zur Entpolitisierung von sozialen Konflikten und schließe bestimmte sozial oder kulturell benachteiligte Gruppen aus dem Diskussionsprozess aus. Die Antwort muss differenziert ausfallen.

Einerseits sind konservierende Tendenzen feststellbar. Das deliberative Modell stellt einen hohen Anspruch an die Beurteilungskompetenz von BürgerInnen, der angesichts professionalisierter Politik und stratifizierter Öffentlichkeit schwer erfüllbar scheint. Einer Befähigung zur fundierten Kritik am politischen Status quo stehen hohe Hürden entgegen. Im schlimmsten Fall können die bestehenden Verhältnisse trotz bestehender demokratiegefährdender sozialer Ungleichheiten stillschweigend legitimiert werden. Des Weiteren lässt sich bei manchen Interpretationen des deliberativen Modells eine konzeptuelle Engführung von Deliberation auf formalistische Kriterien identifizieren. Diese können dazu führen, dass alternative Begründungsformen dem Absolutismus eines formalen Rationalismus geopfert werden. Marginalisierte Perspektiven würden delegitimiert und real existierende Konflikte entpolitisiert. Kulturell vorherrschende Rollenmuster und Bewertungskriterien schlagen auf das deliberative Partizipationsverhalten durch und benachteiligen so sozial unterprivilegierte Gruppen.

Andererseits macht unsere Analyse deutlich, dass diese Tendenzen selbst unter den Ungleichheitsbedingungen gegenwärtiger demokratischer Systeme keineswegs 
zwangsläufig sind. So zeigen theoretische Überlegungen und empirische Studien Wege auf, wie deliberative Praktiken auch gegenüber hegemonialen Diskursen Emanzipationseffekte erzielen und zur Kritik am Status quo befähigen können. Sie demonstrieren, wie die Öffnung gegenüber alternativen Artikulationsformen politischer Positionen und Begründungen (z. B. Storytelling) einen genuin deliberativen Beitrag zu Willensbildungsprozessen liefern und Letztere pluralisieren können. Schließlich belegen sie, wie institutionelle Arrangements die Ausschlussmechanismen neutralisieren und deliberative Prozesse inklusiver gestalten können.

Nimmt man die skizzierten Ausschlussmechanismen ernst, müssen die VertreterInnen der deliberativen Demokratie nach den geeigneten Mitteln fragen, emanzipatorische Potenziale deliberativer Praktiken und Institution zu sichern und ihre konservierenden Tendenzen zu vermeiden. Als unfruchtbar erweist sich dabei das Denken in dichotomen Alternativen. Weder ein „Je mehr Deliberation desto besser“ noch ein „Entweder Deliberation oder Repräsentation“ ist dabei zielführend. Denn Deliberation ist ein soziales Faktum. Nicht nur sind Elemente des deliberativen Modells längst in Form von institutionalisierten Verfahren und von demokratischen Innovationen innerhalb repräsentativer Demokratien etabliert (Curato et al. 2017; Pogrebinschi und Ryan 2018; Schäfer 2017a). Die allgemeinen Vorstellungen der deliberativen Demokratie haben mittlerweile auch eine hohe normative Plausibilität und Attraktivität entfaltet. Es muss also darum gehen, begründete Vermutungen darüber anzustellen, wie das demokratisch-emanzipatorische Potenzial entfaltet und die unerwünschten Wirkungen vermieden werden könnten.

Demokratische Deliberation erscheint vor diesem Hintergrund als unerlässliche, aber nicht hinreichende Bedingung demokratischer Legitimität. Die deliberative Dimension ist deswegen für demokratische Systeme unverzichtbar, weil sie einen kritischen Maßstab für deren Willensbildungsprozesse liefert. Die deliberative Perspektive lenkt dabei die Aufmerksamkeit auf die weitere Demokratisierung von partizipativen und repräsentativen Willensbildungs- und Entscheidungsprozessen. Sie fokussiert auf die demokratische Verbindung von Inklusion und (kommunikativer) Rationalität, die ein rein auf Stimmenaggregation gerichteter Blickwinkel außen vor lässt. Sie ist aber deswegen nicht hinreichend, weil sie nicht allein sicherstellen kann, dass ihre Emanzipationspotenziale nicht durch den Status quo konservierende, entpolitisierende und exkludierende Tendenzen ausgehebelt werden. Deshalb erscheint die komplementäre Einbettung von Deliberation in das demokratische Gesamtsystem als notwendige emanzipatorische Voraussetzung, weil Letzteres den institutionellen Rahmen liefern kann, um die deliberativen Demokratisierungspotenziale zu entfalten und ihre problematischen Mechanismen zu entschärfen. Denn die Wirkungsrichtung deliberativer Prozesse hängt, wie die vorliegende Analyse gezeigt hat, von vielfältigen institutionellen Kontextfaktoren ab. Dazu zählen: Befristungsund Entscheidungsregeln, die Unabhängigkeit der Medien, vorhandene Transmissionsmechanismen zwischen schwachen und starken Öffentlichkeiten, strukturelle Partizipationsgelegenheiten und - nicht zuletzt - kulturell vorherrschende Bewertungshierarchien.

Im besten Fall kann die theoretische und empirische Analyse Anhaltspunkte liefern, wie das Demokratisierungspotenzial deliberativer Prozesse gesichert werden kann. Insbesondere die Tradition des systemischen Denkens innerhalb der delibera- 
tiven Demokratietheorie kann Instrumente liefern, die konservierenden Tendenzen deliberativer Prozesse zu neutralisieren. Doch nicht jedes konservierende Element muss demokratietheoretisch problematisch sein. Verlässliche Verfahren mögen zwar konservierend, aber demokratietauglich sein, weil sie Erwartungssicherheit für alle Parteien und BürgerInnen liefern. Die demokratische Qualität deliberativer Verfahren kann also nicht ausschließlich theoretisch-abstrakt beurteilt werden. Systemische Lösungsansätze müssen daher sowohl selbstreflexiv als auch offen sein für die Perspektive und Kritik der am politischen Prozess Beteiligten.

Funding Open Access funding provided by Projekt DEAL.

Open Access Dieser Artikel wird unter der Creative Commons Namensnennung 4.0 International Lizenz veröffentlicht, welche die Nutzung, Vervielfältigung, Bearbeitung, Verbreitung und Wiedergabe in jeglichem Medium und Format erlaubt, sofern Sie den/die ursprünglichen Autor(en) und die Quelle ordnungsgemäß nennen, einen Link zur Creative Commons Lizenz beifügen und angeben, ob Änderungen vorgenommen wurden.

Die in diesem Artikel enthaltenen Bilder und sonstiges Drittmaterial unterliegen ebenfalls der genannten Creative Commons Lizenz, sofern sich aus der Abbildungslegende nichts anderes ergibt. Sofern das betreffende Material nicht unter der genannten Creative Commons Lizenz steht und die betreffende Handlung nicht nach gesetzlichen Vorschriften erlaubt ist, ist für die oben aufgeführten Weiterverwendungen des Materials die Einwilligung des jeweiligen Rechteinhabers einzuholen.

Weitere Details zur Lizenz entnehmen Sie bitte der Lizenzinformation auf http://creativecommons.org/ licenses/by/4.0/deed.de.

\section{Literatur}

Bächtiger, André, John S. Dryzek, Jane J. Mansbridge, und Mark E. Warren (Hrsg.). 2018. The Oxford handbook of deliberative democracy. Oxford: Oxford University Press.

Bächtiger, André, Simon Niemeyer, Michael Neblo, Marco R. Steenbergen, und Jürg Steiner. 2010. Disentangling diversity in deliberative democracy. Competing theories, their blind spots and complementarities. Journal of Political Philosophy 18(1):32-63.

Benhabib, Seyla. 1996. Toward a deliberative model of democratic legitimacy. In Democracy and difference: contesting the boundaries of the political, Hrsg. Seyla Benhabib, 67-94. Princeton: Princeton University Press.

Bohman, James. 1998. Survey article: the coming of age of deliberative democracy. Journal of Political Philosophy 6(4):400-425.

Boswell, John, Carolyn M. Hendriks, und Selen A. Ercan. 2016. Message received? Examining transmission in deliberative systems. Critical Policy Studies 10(3):263-283.

Buchstein, Hubertus, und Dirk Jörke. 2003. Das Unbehagen an der Demokratietheorie. Leviathan 31(4):470-495.

Burke, Edmund. 1790. Reflections on the revolution in France, and on the proceedings in certain societies in London relative to that event. London: James Dodsley.

Chambers, Simone. 2004. Behind closed doors. Publicity, secrecy, and the quality of deliberation. Journal of Political Philosophy 12(4):389-410.

Chambers, Simone. 2009. Rhetoric and the public sphere: has deliberative democracy abandoned mass democracy? Political Theory 37(3):323-350.

Cohen, Joshua. 1998. Democracy and liberty. Deliberative Democracy 1:185-231.

Crouch, Colin. 2004. Post-democracy. Cambridge: Polity.

Curato, Nicole, und Jonathan Corpus Ong. 2014. Inclusion as deliberative agency: the selective representation of poor women in debates and documentaries about reproductive health. Television \& New Media 16(6):576-594.

Curato, Nicole, John S. Dryzek, Selen A. Ercan, Carolyn M. Hendriks, und Simon Niemeyer. 2017. Twelve key findings in deliberative democracy research. Daedalus 146(3):28-38. 
Druckman, James, und Kjersten Nelson. 2003. Framing and deliberation: how citizens' conversations limit elite influence. American Journal of Political Science 47(4):729-745.

Dryzek, John S. 2000. Deliberative democracy and beyond: liberals, critics, contestations. Oxford: Oxford University Press.

Dryzek, John S. 2010. Foundations and frontiers of deliberative governance. Oxford: Oxford University Press.

Elster, Jon. 1998. Introduction. In Deliberative Democracy, Hrsg. Jon Elster, 1-18. Cambridge, New York: Cambridge University Press.

Elstub, Stephen, und Peter McLaverty (Hrsg.). 2014. Deliberative democracy. Issues and cases. Edinburgh: Edinburgh University Press.

Esterling, Kevin M., Archon Fung, und Taeku Lee. 2015. How much disagreement is good for democratic deliberation? Political Communication 32(4):529-551.

Fishkin, James S., und Robert C. Luskin. 2005. Experimenting with a democratic ideal: deliberative polling and public opinion. Acta politica 40(3):284-298.

Fishkin, James S., und Jane Mansbridge. 2017. Introduction. Daedalus 146(3):6-13.

Flügel-Martinsen, Oliver, Daniel Gaus, Tanja Hitzel-Cassagnes, und Franziska Martinsen (Hrsg.). 2014. Deliberative Kritik - Kritik der Deliberation. Festschrift für Rainer Schmalz-Bruns. Wiesbaden: Springer VS.

Fraile, Marta. 2014. Does deliberation contribute to decreasing the gender gap in knowledge? European Union Politics 15(3):372-388.

Fung, Archon. 2005. Deliberation before the revolution. Toward an ethics of deliberative democracy in an unjust world. Political Theory 33(3):397-419.

Gerber, Marlène. 2015. Equal partners in dialogue? Participation equality in a transnational deliberative poll (Europolis). Political Studies 63(1):110-130.

Goodin, Robert. 2018. If deliberation is everything, maybe it's nothing. In The Oxford handbook of deliberative democracy, Hrsg. André Bächtiger, John S. Dryzek, Jane J. Mansbridge, und Mark E. Warren, 882-899. Oxford: Oxford University Press.

Greiffenhagen, Martin. 2010. Konservativ, Konservatismus. In Historisches Wörterbuch der Philosophie, Bd. 4, Hrsg. Joachim Ritter, Karlfried Gründer, und Gottfried Gabriel, 980-985. Basel: Schwabe.

Gutmann, Amy, und Dennis Thompson. 1996. Democracy and disagreement: why moral conflict cannot be avoided in politics, and what can be done about it. Cambridge: Belknap Press.

Gutmann, Amy, und Dennis Thompson. 2004. Why deliberative democracy? Princeton: Princeton University Press.

Habermas, Jürgen. 1990. Strukturwandel der Öffentlichkeit. Untersuchungen zu einer Kategorie der bürgerlichen Gesellschaft. Frankfurt am Main: Suhrkamp.

Habermas, Jürgen. 1994. Faktizität und Geltung. Beiträge zur Diskurstheorie des Rechts und des demokratischen Rechtsstaats. Frankfurt am Main: Suhrkamp.

Habermas, Jürgen. 1996. Die Einbeziehung des Anderen. Studien zur politischen Theorie. Frankfurt am Main: Suhrkamp.

Habermas, Jürgen. 2008. Hat die Demokratie noch eine epistemische Dimension? Empirische Forschung und normative Theorie. In Ach, Europa, Hrsg. Jürgen Habermas, 138-191. Frankfurt am Main: Suhrkamp.

Hamilton, Andy. 2016. Conservatism. In The Stanford Encyclopedia of Philosophy, Hrsg. Edward N. Zalta. http://plato.stanford.edu/archives/fall2016/entries/conservatism/. Zugegriffen: 5. Nov. 2016.

von Hayek, Friedrich August. 1971. Die Verfassung der Freiheit. Tübingen: Mohr.

Hendriks, Carolyn M. 2016. Coupling citizens and elites in deliberative systems: the role of institutional design. European Journal of Political Research 55(1):43-60.

Holdo, Markus. 2019. Meta-deliberation: everyday acts of critical reflection in deliberative systems. Politics. https://doi.org/10.1177/0263395719837914.

Karpowitz, Christopher F., Tali Mendelberg, und L.E.E. Shaker. 2012. Gender inequality in deliberative participation. American Political Science Review 106(3):533-547.

Lafont, Christina. 2015. Deliberation, participation, and democratic legitimacy: should deliberative minipublics shape public policy? The Journal of Political Philosophy 23(1):40-63.

Landwehr, Claudia. 2015. Democratic meta-deliberation. Towards reflective institutional design. Political Studies 63(1_suppl):38-54.

Landwehr, Claudia, und Rainer Schmalz-Bruns. 2014. Deliberative Demokratie in der Diskussion. Herausforderungen, Bewährungsproben, Kritik. Baden-Baden: Nomos.

Linz, Juan J. 1998. Democracy‘s time constraints. International Political Science Review 19(1):19-37. 
Lomax Cook, Fay, Michael X. Delli Carpini, und Lawrence R. Jacobs. 2007. Who deliberates? Discursive participation in america: deliberation, participation and democracy. In Can the people govern? Deliberation, participation and democracy, Hrsg. Shan W. Rosenberg, 25-40. New York: Macmillan.

Mansbridge, Jane. 1999. Everyday talk in the deliberative system. In Deliberative politics: essays on democracy and disagreement, Hrsg. Stephen Macedo, 211-242. Oxford, New York: Oxford University Press.

Mansbridge, Jane, James Bohman, Simone Chambers, Thomas Christiano, Archon Fung, John Parkinson, Dennis F. Thompson, und Mark E. Warren. 2012. A systemic approach to deliberative democracy. In Deliberative systems. Deliberative democracy at the large scale, Hrsg. John Parkinson, 1-26. Cambridge, New York: Cambridge University Press.

Mau, Steffen, und Nadine M. Schöneck (Hrsg.). 2015. (Un-)gerechte (Un-)gleichheiten. Berlin: Suhrkamp.

McLaverty, Peter. 2014. Inequality and deliberative democracy. In Deliberative democracy: issues and cases, Hrsg. Stephen Elstub, Peter McLaverty, 34-49. Edinburgh: Edinburgh University Press.

Mendelberg, Tali, Christopher F. Karpowitz, und J. Baxter Oliphant. 2014. Gender inequality in deliberation: unpacking the black box of interaction. Perspectives on Politics 12(1):18-44.

Merkel, Wolfgang. 2014. Is Capitalism compatible with Democracy? Zeitschrift für vergleichende Politikwissenschaft 8(2):109-128.

Merkel, Wolfgang. 2015. Demokratie und Krise. Zum schwierigen Verhältnis von Theorie und Empirie. Wiesbaden: Springer VS.

Merkel, Wolfgang, und Andreas Schäfer. 2015. Zeit und Demokratie: Ist demokratische Politik zu langsam? In Sonderband Leviathan Zeit der Politik, Demokratisches Regieren in einer beschleunigten Welt, Bd. 30, Hrsg. Holger Straßheim, Tom Ulbricht, 217-237.

Mouffe, Chantal. 2005. The democratic paradox. London: Verso.

Mouffe, Chantal. 2007. Über das Politische. Wider die kosmopolitische Illusion. Frankfurt am Main: Suhrkamp.

Müller, Jan-Werner. 2006. Comprehending conservatism: a new framework for analysis. Journal of Political Ideologies 11(3):359-365.

Neblo, Michael A., Kevin M. Esterling, Ryan P. Kennedy, David M. Lazer, und Anand E. Sokhey. 2010. Who wants to deliberate-and why? American Political Science Review 104(3):566-583.

Niemeyer, Simon. 2011. The emancipatory effect of deliberation: empirical lessons from mini-publics. Politics \& Society 39(1):103-140.

Niesen, Peter. 2014. Was heißt Deliberation? Eine theoriegeschichtliche Betrachtung. In Deliberative Kritik-Kritik der Deliberation, Hrsg. Oliver Flügel-Martinsen, Daniel Gaus, Tanja Hitzel-Cassagnes, und Franziska Martinsen, 49-71. Wiesbaden: Springer VS.

Owen, David, und Graham Smith. 2015. Survey article. Deliberation, democracy, and the systemic turn. Journal of Political Philosophy 23(2):213-234.

Peters, Bernhard. 2007. Der Sinn von Öffentlichkeit. Frankfurt am Main: Suhrkamp.

Pogrebinschi, Thamy, und Matt Ryan. 2018. Moving beyond input legitimacy: when do democratic innovations affect policymaking? European Journal of Political Research 57(1):135-152.

Pogrebinschi, Thamy, und David Samuels. 2014. The impact of participatory democracy. Evidence from Brazil's national public conferences. Comparative Politics 46(3):313-332.

Polletta, Francesca, und John Lee. 2006. Is telling stories good for democracy? Rhetoric in public deliberation after 9/11. American Sociological Review 71(5):699-723.

Przeworski, Adam. 1998. Deliberation and Ideological Domination. In Deliberative Democracy, Hrsg. Jon Elster, 140-160. Cambridge, New York: Cambridge University Press.

Rosenberg, Shawn W. 2014. Citizen competence and the psychology of deliberation. In Deliberative democracy: issues and cases, Hrsg. Stephen Elstub, Peter McLaverty, 98-117. Edinburgh: University Press.

Sanders, Lynn M. 1997. Against deliberation. Political Theory 25(3):347-376.

Schäfer, Andreas. 2017a. Zwischen Repräsentation und Diskurs. Zur Rolle von Deliberation im parlamentarischen Entscheidungsprozess. Wiesbaden: Springer.

Schäfer, Andreas. 2017b. Deliberation in representative institutions: an analytical framework for a systemic approach. Australian Journal of Political Science 52(3):419-435.

Shapiro, Ian. 1999. Enough of deliberation. Politics is about interests and power. In Deliberative politics. Essays on democracy and disagreement, Hrsg. Stephen Macedo, 28-38. New York: Oxford University Press.

Shapiro, Ian. 2017. Collusion in restraint of democracy: against political deliberation. Daedalus 146(3):77-84. 
Thaa, Winfried. 2007. Informalisierung und Kognitivierung politischer Repräsentation in deliberativen Demokratietheorien. In Inklusion durch Repräsentation, Hrsg. Winfried Thaa, 85-108. Baden-Baden: Nomos.

Tully, James. 2004. Recognition and dialogue: the emergence of a new field. Critical Review of International Social and Political Philosophy 7(3):84-106.

Urbinati, Nadia. 2000. Representation as advocacy: a study of democratic deliberation. Political Theory 28(6):758-786.

Urbinati, Nadia. 2010. Unpolitical democracy. Political Theory 38(1):65-92.

Vierhaus, Rudolf. 1995. Konservativ, Konservatismus. In Geschichtliche Grundbegriffe, Hrsg. Otto Brunner, Werner Conze, und Reinhart Koselleck, 531-565. Stuttgart: Klett-Cotta.

Warren, Mark E. 2017. A problem-based approach to democratic theory. American Political Science Review 111(1):39-53.

White, Jonathan, und Lea Ypi. 2011. On Partisan Political Justification. American Political Science Review 105(2):381-396.

Young, Iris M. 2000. Inclusion and democracy. Oxford, New York: Oxford University Press.

Young, Iris M. 2001. Activist Challenges to Deliberative Democracy. Political Theory 29(5):670-690. 